\title{
ECG baseline wander removal with recovery of the isoelectric level
}

\author{
Antonio Fasano ${ }^{1}$, Valeria Villani ${ }^{2}$ \\ ${ }^{1}$ Università Campus Bio-Medico di Roma, Rome, Italy \\ ${ }^{2}$ Università di Modena e Reggio Emilia, Reggio Emilia, Italy
}

\begin{abstract}
Baseline wander removal is an unavoidable step in ECG signal processing. The in-band nature of this noise makes its removal difficult without affecting the ECG. Many approaches have been proposed in the literature. Among them, cubic spline interpolation is the only one able to recover the isoelectric level in the detrended signal. However, it exhibits poor detrending performance. In this paper we extend our recent approach based on the notion of quadratic variation reduction, to address the problem of recovery of the isoelectric level. This is achieved by constraining the amplitude of few fiducial points to lie on isoelectric segments. Conversely to cubic spline interpolation, which requires a fiducial point for each beat, the proposed approach requires very few points: as few as one single point is sufficient. Simulation results show that the proposed approach largely outperforms cubic spline interpolation, being very effective in removing baseline wander and recovering the isoelectric level, while preserving ECG morphology.
\end{abstract}

\section{Introduction}

Baseline wander is a kind of noise that affects all bioelectrical signals [1,2]. In ECG recordings it is caused by patient's respiration, perspiration, body movements, skinelectrode interface, and varying impedance between electrodes and skin due to poor electrode contact [1]. It is ubiquitous in all electrocardiographic devices and its removal is an unavoidable step in ECG signal processing [3,4]. Unfortunately, baseline wander and ECG have overlapping bands in the low-frequency region of the spectrum. Hence, its removal is difficult without affecting the ECG, in particular the ST segment [1-3], thus spoiling relevant clinical information [5].

Given the critical role of baseline wander removal for ECG signals, several solutions have been proposed to tackle this problem. The simplest approach is high-pass filtering $[3,6]$. Other common techniques are: adaptive and median filtering $[7,8]$, polynomial fitting, typically cubic splines [9], wavelet decomposition and detrending by quadratic variation reduction (QVR), which we have recently proposed [10]. Among them, cubic spline interpolation (CSI) [9] is the only method that is able to recover the isoelectric level in the detrended signal. However, it exhibits poor detrending performance $[1,11]$.

Although the isoelectric level contains negligible information per se [1], it is the global point of reference for the ECG's amplitude, i.e., the amplitude of any wave or segment (e.g., ST segment) is measured with reference to the isoelectric level [1,2]. Since even small deviations from the isoelectric level are significant markers of cardiac abnormality, the correct measurement of the isoelectric line is crucial [2]. However, it is prone to noise and its estimate may result in significant errors [2]. To tackle this issue, restoring the isoelectric level during signal preprocessing, specifically when removing baseline wander, might be beneficial to subsequent analysis.

In this paper, we extend our recent approach to detrending by QVR, which outperforms state-of-the-art algorithms $[10,11]$. However, it does not recover the isoelectric level in the detrended signal. To overcome this limitation, we introduce additional constraints in the optimization problem and constrain the amplitude of few fiducial points to lie on isoelectric segments. This additional information improves the estimation of baseline wander. Conversely to CSI, which needs a fiducial point for each beat, the proposed algorithm requires very few points and even a single knot is enough. Results show that the approach outperforms CSI in estimating baseline wander and recovering the isoelectric level, while preserving ECG morphology.

The paper is organized as follows. In Section 2 the notion of quadratic variation is introduced and the rationale behind the proposed approach is described. Sections 3 and 4 follow with simulation results and conclusions.

\section{Baseline wander removal with recov- ery of the isoelectric level}

In this section, we adopt the following notation: $\widetilde{z}$ is the vector collecting $n$ samples of an ECG record, which is affected by baseline wander, $\boldsymbol{x}$ is the vector of estimated 
baseline, and $\boldsymbol{z}=\widetilde{\boldsymbol{z}}-\boldsymbol{x}$ is the corresponding ECG vector after detrending.

Let us start by recalling the CSI method [9]. It fits a cubic polynomial to a set of representative points of the ECG, namely knots, with one knot for each beat. Let $\mathcal{I}_{\text {iso }}^{\text {CSI }} \subseteq\{1, \ldots, n\}$ be the set of indices (abscissae) of the knots for CSI: its cardinality $\left|\mathcal{I}_{\text {iso }}^{\mathrm{CSI}}\right|$ equates the number of beats. For each $k \in \mathcal{I}_{\text {iso }}^{\text {CSI }}$ the corresponding level (ordinate) of the knot is denoted by $g_{k} \in \mathbb{R}$. The collection of pairs $\left(k, g_{k}\right)$, with $k \in \mathcal{I}_{\text {iso }}^{\mathrm{CSI}}$, is the set of knots and constitutes the a priori information needed for CSI to work properly.

Conversely, the algorithm proposed in this paper needs much less information to recover the isoelectric level: any subset of knots, even with only one element, is enough. So, denote by $\mathcal{I}_{\text {iso }} \subseteq \mathcal{I}_{\text {iso }}^{\text {CSI }}$ the set of indices of knots used by the proposed algorithm and let $n_{\text {iso }}=\left|\mathcal{I}_{\text {iso }}\right|$ its size. In general $n_{\text {iso }} \ll\left|\mathcal{I}_{\text {iso }}^{\text {CSI }}\right|$, with $n_{\text {iso }}=1$ being perfectly ok, as the numerical results of Section 3 show. Let $\boldsymbol{g}_{\text {iso }}$ be

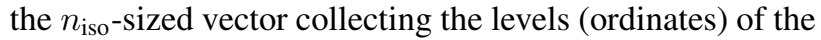
knots indexed by $\mathcal{I}_{\text {iso }}$. Denote by $\boldsymbol{S}_{0}$ the $n_{\text {iso }} \times n$ matrix obtained from the $n \times n$ identity matrix $\boldsymbol{I}_{n}$ by selecting the rows whose indices belong to $\mathcal{I}_{\text {iso }}$, and by $\boldsymbol{S}_{1}$ the complementary $\left(n-n_{\text {iso }}\right) \times n$ matrix obtained from $\boldsymbol{I}_{n}$ by removing the rows whose indices belong to $\mathcal{I}_{\text {iso }}$. With these positions, for any vector $\boldsymbol{x} \in \mathbb{R}^{n}, \boldsymbol{S}_{0} \boldsymbol{x}$ is the subvector that collects all the entries of $\boldsymbol{x}$ whose indices belong to $\mathcal{I}_{\text {iso }}$. Constraining the baseline to pass through the knots amounts to impose $\boldsymbol{S}_{0} \boldsymbol{x}=\boldsymbol{g}_{\text {iso }}$.

Baseline wander noise is an additive low "variability" component affecting the measured ECG. Thus, provided that we introduce a suitable index of "variability", baseline can be estimated searching for the low variability component closest, in some sense, to the measured ECG. With this purpose, in [10] we introduce the quadratic variation as a measure of "variability".

Given a vector $\boldsymbol{x}=\left[x_{1} \cdots x_{n}\right]^{\mathrm{T}} \in \mathbb{R}^{n}$, the quadratic variation of $\boldsymbol{x}$, namely $[\boldsymbol{x}]$, is defined as

$$
[\boldsymbol{x}] \doteq \sum_{k=1}^{n-1}\left(x_{k}-x_{k+1}\right)^{2}=\|\boldsymbol{D} \boldsymbol{x}\|^{2}
$$

where $\|\cdot\|$ denotes the Euclidean norm and $\boldsymbol{D}$ is the $(n-$ 1) $\times n$ matrix with entries

$$
\boldsymbol{D}_{i j}=\delta_{i, j}-\delta_{i+1, j}
$$

where $\delta_{i, j}$ is the Kronecker delta. It can be proven that the quadratic variation is a consistent measure of variability [10].

Baseline wander can be estimated as the component closest to the observed ECG that has reduced quadratic variation, and is constrained to pass through the knots indexed by $\mathcal{I}_{\text {iso }}$ to recover the isoelectric level. It can be

\footnotetext{
${ }^{1}$ Low with respect to ECG "variability".
}

estimated by solving the following optimization problem, which is a generalization of [10]

$$
\left\{\begin{array}{cl}
\underset{\boldsymbol{x} \in \mathbb{R}^{n}}{\operatorname{minimize}} & \|\boldsymbol{x}-\widetilde{\boldsymbol{z}}\|^{2} \\
\text { subject to } & {[\boldsymbol{x}] \leq \rho} \\
& \boldsymbol{S}_{0} \boldsymbol{x}=\boldsymbol{g}_{\text {iso }}
\end{array}\right.
$$

where the first constraint with $\rho \geq 0$ controls the quadratic variation of the estimated baseline $\boldsymbol{x}$, and the second forces the estimated baseline to pass through the knots indexed by $\mathcal{I}_{\text {iso. }}$.

Let us consider (3) in more detail. It is a convex optimization problem, since both the objective function and the constraints are convex. As a consequence, any locally optimal point is also globally optimal [12]. Moreover, since the objective function is strictly convex and the problem is feasible, the solution exists and is unique. It is possible to prove that the solution to (3) is given by

$$
\begin{aligned}
\boldsymbol{x}=\boldsymbol{S}_{0}^{T} \boldsymbol{g}_{\mathrm{iso}}+\boldsymbol{S}_{1}^{T}[ & \left.\boldsymbol{I}_{n-n_{\text {iso }}}+\lambda \boldsymbol{S}_{1} \boldsymbol{D}^{T} \boldsymbol{D} \boldsymbol{S}_{1}^{T}\right]^{-1} \\
& \times \boldsymbol{S}_{1}\left(\widetilde{\boldsymbol{z}}-\lambda \boldsymbol{D}^{T} \boldsymbol{D} \boldsymbol{S}_{0}^{T} \boldsymbol{g}_{\text {iso }}\right)
\end{aligned}
$$

where $\boldsymbol{I}_{n-n_{\text {iso }}}$ denotes the $\left(n-n_{\text {iso }}\right) \times\left(n-n_{\text {iso }}\right)$ identity matrix, and $\lambda$ is a nonnegative parameter that controls the quadratic variation of the solution $\boldsymbol{x}$, i.e., the degree of variability of the estimated baseline. The parameter $\lambda$ is in one-to-one correspondence with $\rho$. Note that we do not need to care about $\rho$, since only $\lambda$ is used in (4) as the controlling parameter. The detrended signal $z$ is obtained by subtracting the estimated baseline

$$
z=\widetilde{z}-x
$$

It is possible to prove that estimating baseline using (4) is very fast, since its complexity is $O(n)$, i.e., linear in the size of the vector $\widetilde{z}$ to detrend. Moreover, it is worth remarking that to recover the isoelectric level it is sufficient to choose one knot only, i.e., $n_{\text {iso }}=1$. In the following we will refer to baseline wander removal using (5) and (4) with the acronym QVRi.

\section{Simulation results}

We considered a portion of the ECG record mitdb/118 from the MIT-BIH Arrhythmia Database [13] from PhysioNet [14]. It is a two-channel recording acquired at a sampling frequency of $360 \mathrm{~Hz}$ with 11 -bit resolution and is affected by measurement noise and negligible baseline wander. Such a reference record, denoted in the following by $\boldsymbol{z}_{0}$, was further corrupted with baseline wander, namely $\boldsymbol{b}$, and random level shift, namely $\boldsymbol{s}$. Baseline wander was either extracted from the record nstdb/bw from 

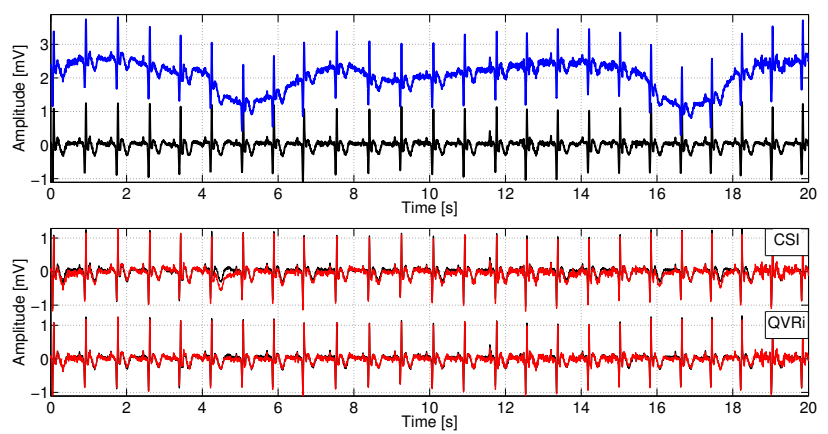

Figure 1. Top: real ECG ( $\boldsymbol{z}_{0}$, black) corrupted with baseline wander ( $\widetilde{z}$, blue). Bottom: ECG detrended using CSI and QVRi $\left(\boldsymbol{z}\right.$, red), and reference ECG $\left(\boldsymbol{z}_{0}\right.$, black).

the MIT-BIH Noise Stress Test Database [14, 15], or rendered as Gaussian white noise with zero mean and variance $\sigma^{2}=6.25$ low-pass filtered with bandwidth $0.8 \mathrm{~Hz}$, for the statistical analysis. The random level shift was rendered as a constant vector $s=U[1 \ldots 1]^{T}$, where $U$ is a random variable with uniform distribution in the interval $[-5,5]$. The corrupted signal, denoted by $\widetilde{z}=\boldsymbol{z}_{0}+\boldsymbol{b}+\boldsymbol{s}$, is reported (blue) in the upper panel of Fig. 1 together with the original record $z_{0}$ (black). In this case baseline wander was extracted from the record nstdb/bw. In the lower panel of Fig. 1 we report the reference ECG $\boldsymbol{z}_{0}$ (black) and the corresponding records $z$ (red) detrended using CSI (upper half) and the proposed algorithm (lower half) with $\lambda$ roughly set to $3 \times 10^{3}$. Lags relative to PQ isoelectric segments were roughly identified by visual inspection on the reference record $z_{0}$; the corresponding isoelectric levels were determined averaging the record $z$ in a window of 9 samples centered around each lag [9]. A visual comparison highlights the better performance of the proposed approach, which manages to effectively detrend the ECG record recovering the isoelectric level.

For a statistical assessment of the performance, we considered 200 independent realizations of synthetic baseline wander, rendered as described before, and compared our algorithm with CSI. The quality of detrending was quantified through the following error

$$
\varepsilon_{i}\left(\operatorname{alg}_{k}\right)=\frac{\left\|\boldsymbol{z}_{i}\left(\operatorname{alg}_{k}\right)-\boldsymbol{z}_{0}\right\|^{2}}{\left\|\boldsymbol{z}_{0}\right\|^{2}} \quad i=1, \ldots, 200
$$

where $\boldsymbol{z}_{i}\left(\operatorname{alg}_{k}\right)$ is the record detrended using algorithm $\operatorname{alg}_{k} \in\{\mathrm{QVRi}, \mathrm{CSI}\}$. Performance is measured in terms of the empirical distribution function of the error (6), namely

$$
\hat{F}_{\operatorname{alg}_{k}}(\epsilon)=\frac{1}{N} \sum_{i=1}^{N} \chi_{(-\infty, \epsilon]}\left[\varepsilon_{i}\left(\operatorname{alg}_{k}\right)\right]
$$

where $\chi_{E}[\cdot]$ denotes the indicator function of the set $E$, and $N$ is the number of generated trend realizations

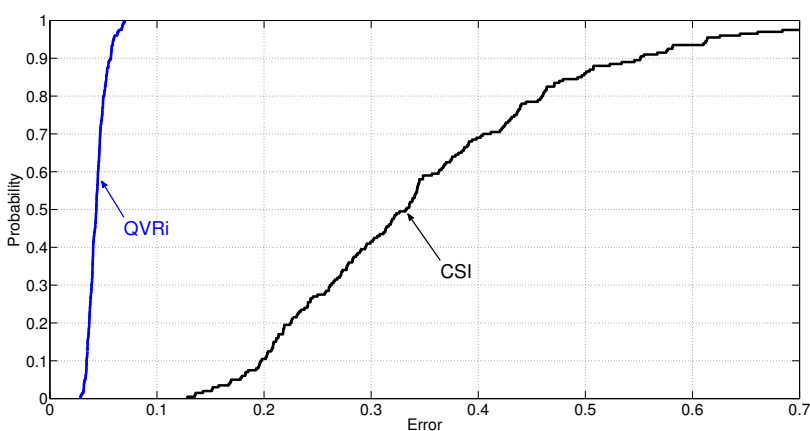

Figure 2. Empirical distribution functions of the error (6) for QVRi (blue) and CSI (black).

$(N=200)$. The empirical distribution function (7) provides a complete statistical description of the performance of each algorithm, as opposed to the mean, the variance, or the median, which give only partial information. Indeed, empirical distribution functions allow us to compare algorithms over the full range of errors taking account of error relative frequencies. Moreover, they are useful for characterizing algorithms that have uniformly better performance (in statistical sense) over the whole range of errors. To this end, denoting by $\hat{F}_{A}(\epsilon)$ and $\hat{F}_{B}(\epsilon)$ the empirical distribution functions of errors, relative to algorithms $A$ and $B$ respectively, we introduce the following

Definition 1. Algorithm A is statistically uniformly better than algorithm $B$ if

$$
\hat{F}_{A}(\epsilon)>\hat{F}_{B}(\epsilon), \quad \forall \epsilon \in \mathcal{E}_{A B}
$$

with $\mathcal{E}_{A B}=\left\{\varepsilon \in \mathbb{R} \mid 0<\hat{F}_{A}(\varepsilon)+\hat{F}_{B}(\varepsilon)<2\right\}$.

Set $\mathcal{E}_{A B}$ consists of all real numbers except those for which $\hat{F}_{A}(\epsilon)=\hat{F}_{B}(\epsilon) \in\{0,1\}$, which are not relevant for the comparison. If an algorithm is statistically uniformly better than another, then it exhibits lower errors with higher probability. Being statistically uniformly better is a strong property and, as we will show below, QVRi is statistically uniformly better than CSI.

Fig. 2 reports the empirical distribution functions of error (6) for QVRi and CSI. The parameter $\lambda$ was roughly set to $3 \times 10^{3}$. The same knots were used for both the algorithms. As Fig. 2 highlights, detrending by QVRi largely outperform CSI, since it returns lower errors with higher probability. In particular, QVRi is statistically uniformly better than CSI. Moreover, it is worth noting that the gap between the empirical distribution functions of the two algorithms is remarkably high. This is a measure of the performance margin that QVRi has over CSI.

Finally, we analyzed how the performance of QVRi is affected by the number of knots. In particular, in Fig. 3 we reported the empirical distribution functions of error (6) 


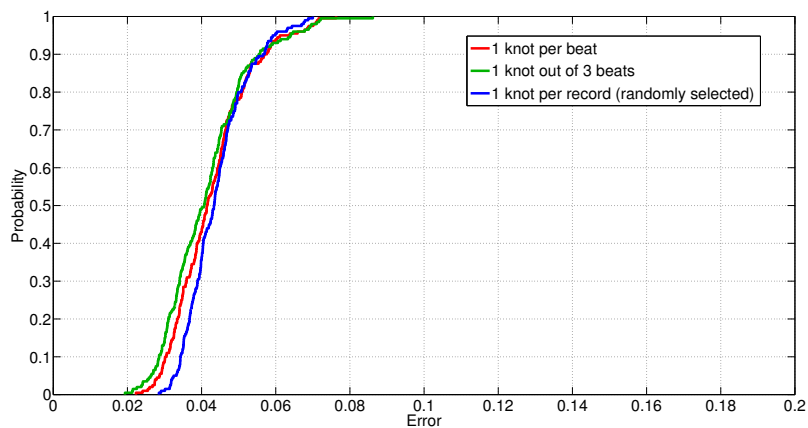

Figure 3. Empirical distribution functions of error (6) for QVRi with different numbers of knots.

relative to the the following cases: i) one knot for each beat (blue) (the same blu curve of Fig. 7); ii) one knot out of three beats (red); iii) only one knot for the whole record, randomly selected (green). As Fig. 3 highlights, the curves are nearly superimposed, thus proving that the performance of QVRi is (practically) not affected by the number of knots and their location. Conversely, the performance of CSI is critically dependent on the number of knots and the accuracy of knot determination $[1,2]$.

\section{Conclusions}

In this paper we consider the problem of baseline wander removal for ECG signals, with recovery of the isoelectric level. The proposed method, namely QVRi, is an extension of our recent approach based on quadratic variation reduction. Additional constraints are introduced in the optimization problem to constrain the amplitude of few fiducial points of the estimated baseline to lie on isoelectric segments. Performance is assessed through a statistical analysis based on the empirical distribution function of the reconstruction error: this allows us to compare algorithms over the full range of errors taking account of error relative frequencies. Numerical results confirm the effectiveness of our approach. It outperforms cubic spline interpolation, which is the only method in the literature able to recover the isoelectric level. In particular, QVRi is statistically uniformly better than cubic spline interpolation. Moreover, conversely to cubic spline interpolation, which requires a fiducial point for each beat, the proposed method works (very) well with one single point. Finally, the proposed algorithm compares favorably also in terms of computational complexity, which is linear in the size of the signal to detrend.

\section{References}

[1] Sörnmo L, Laguna P. Bioelectrical Signal Processing in Cardiac and Neurological Applications. Elsevier Academic
Press, 2005.

[2] Clifford GD, Azuaje F, McSharry P (eds.). Advanced Methods and Tools for ECG Data Analysis. Artech House, Inc., 2006.

[3] Kligfield P, Gettes LS, Bailey JJ, Childers R, Deal BJ, Hancock EW, van Herpen G, Kors JA, Macfarlane P, Mirvis DM, Pahlm O, Rautaharju P, Wagner GS. Recommendations for the standardization and interpretation of the electrocardiogram: Part I: The electrocardiogram and its technologythe electrocardiogram and its technology. Circulation 2007;115:1306-1324.

[4] Gregg RE, Zhou SH, Lindauer JM, Helfenbein ED, Giuliano KK. What is inside the electrocardiograph? J Electrocardiol 2008;41:8-14.

[5] Brewer AJ, Lane ES, Ross P, Hachwa B. Misdiagnosis of perioperative myocardial ischemia: The effects of electrocardiogram filtering. Anesth Analg 2006;103(6):16321634.

[6] Van Alsté JA, Schilder TS. Removal of base-line wander and power-line interference from the ECG by an efficient FIR filter with a reduced number of taps. IEEE Trans Biomed Eng 1985;32(12):1052-1060.

[7] Thakor NV, Zhu YS. Applications of adaptive filtering to ECG analysis: Noise cancellation and arrhythmia detection. IEEE Trans Biomed Eng 1991;38(8):785-794.

[8] Hiasat AA, Al-Ibrahim MM, Gharaibeh KM. Design and implementation of a new efficient median filtering algorithm. IEE Proc Vis Image Signal Process 1999; 146(5):273-278.

[9] Meyer CR, Keiser HN. Electrocardiogram baseline noise estimation and removal using cubic splines and statespace computation techniques. Comp Biomed Res 1977; 10(5):459-470.

[10] Fasano A, Villani V. Baseline wander removal for bioelectrical signals by quadratic variation reduction. Signal Processing 2014;99:48-57. ISSN 0165-1684.

[11] Fasano A, Villani V. ECG baseline wander removal by QVR preserving the ST segment. 8th Conf European Study Group on Cardiac Oscillations ESGCO 2014 2014;

[12] Boyd S, Vandenberghe L. Convex Optimization. Cambridge University Press, March 2004.

[13] Moody GB, Mark RG. The impact of the MIT-BIH arrhythmia database. IEEE Trans Biomed Eng 2001;20(3):45-50.

[14] Goldberger AL, Amaral LAN, Glass L, Hausdorff JM, Ivanov PC, Mark RG, Mietus JE, Moody GB, Peng CK, Stanley HE. PhysioBank, PhysioToolkit, and PhysioNet: Components of a new research resource for complex physiologic signals. Circulation 2000;101(23):e215-e220.

[15] Moody GB, Muldrow W, Mark RG. A noise stress test for arrhythmia detectors. Computers in Cardiology 1984; 11:381-384.

Address for correspondence:

Antonio Fasano

Università Campus Bio-Medico di Roma

via Álvaro del Portillo, 21

00128 Rome, Italy

E-mail: a.fasano@unicampus.it 